Revista de Matemática: Teoría y Aplicaciones 2003 10(1-2) : 187-199

CIMPA - UCR - CCSS ISSN: 1409-2433

\title{
ANÁLISIS DEL FACTOR BETA $(\beta)$ EN EL MODELO DE AMPLITUD
}

\author{
José G. Hernández R.* María J. García G. ${ }^{\dagger}$
}

Recibido: 17 Jun 2003

\begin{abstract}
Resumen
En un reciente trabajo se presentó un modelo para ayudar a la toma de decisiones bajo incertidumbre, cuya principal característica es que dicho modelo, más allá de lo que hacen los modelos tradicionales, toma en cuenta la dispersión.

Dicho modelo, el Modelo de Amplitud (EMA), consiste básicamente en evaluar los valores esperados de las alternativas y corregir estos valores al tomar en cuenta la dispersión de los pagos.

En particular se trabajo con la amplitud, debido a su sencillez y facilidad de aplicación. Al hacer uso de la dispersión en el modelo fue necesaria la construcción de un factor beta $(\beta)$, el cual se usa precisamente para castigar la amplitud.

Para validar el modelo se probó con algunos problemas particulares en su mayoría, tomados de la literatura especializada, quedando sentada su aplicabilidad, pero a la vez se notó que el rango de variación del factor beta $(\beta)$, no pareciera tener sentido que fuese desde cero hasta uno, como se estableció en un principio, por lo cual como recomendación de dicha investigación se propuso, que se analizara con mayor profundidad el rango de variación de beta $(\beta)$.

Con este trabajo de investigación se presenta el resultado de este estudio, para el cual se siguió el método científico aplicado a la investigación de operaciones, donde primero se definió el problema, lo que se puede transcribir como los objetivos de la investigación: Analizar la variabilidad del factor beta $(\beta)$, en las aplicaciones de El Modelo de Amplitud (EMA), para que los resultados obtenidos con su aplicación no pierdan sentido físico.
\end{abstract}

Palabras clave: Toma de decisiones, Toma de decisiones bajo incertidumbre, Dispersión, El Modelo de Amplitud (EMA), Factor beta $(\beta)$.

\footnotetext{
${ }^{*}$ Universidad Metropolitana, Escuela de Ingeniería de Sistemas. Caracas Venezuela. E-Mail: jhernandez@unimet.edu.ve

${ }^{\dagger}$ Minimax Consultores C.A.. Gerencia General. Apartado 78239. Caracas 1074 Venezuela. E-Mail: MJGarcia.Minimax@mercaglob.com
} 


\begin{abstract}
In a recent work a model appeared to help to the decision making under uncertainty, whose main characteristic is that this model, beyond which they make the models traditional, takes into account the dispersion. This model, The Amplitude Model (TAM) [El Modelo de Amplitud (EMA)], consists basically of evaluating the expected values of the alternatives and correcting these values when taking into account the dispersion of the payments.

It is working with the amplitude, due to its simplicity and facility of application. When doing use of the dispersion in the model was necessary the construction of a factor beta $(\beta)$, which is used indeed to punish the amplitude. In order to validate the model it was proven with some particular problems in his majority, taken from specialized Literature, being seated his applicability, but simultaneously the rank of variation of the factor noticed that beta $(\beta)$, did not seem to have sense that was from zero to one, as it settled down at first, thus as recommendation of this research emerge out, that will analyze with greater depth the rank of beta variation $(\beta)$.

With this research the result of this study appears, for which the scientific method applied to research operations, where first the problem was defined, which can be transcribed like the objectives of the investigation: To analyze the variability of the factor beta $(\beta)$, in the applications of The Amplitude Model (TAM), so that the results obtained with their application do not lose physical sense.
\end{abstract}

Keywords: Decision making, Decision making under uncertainty, Dispersion, The Amplitude Model (TAM), Factor beta $(\beta)$.

Mathematics Subject Classification: 90C05, 90-08.

\title{
1 Introducción
}

En un trabajo recientemente presentado se propuso una nueva metodología que ayude a resolver problemas de toma de decisiones bajo incertidumbre. Dicha metodología a la cual se le dio el nombre de El Modelo de Amplitud (EMA), no pretende sustituir a la existentes, sino contribuir a una toma de decisiones más adecuada, cuando, por condiciones particulares del problema, los valores esperados de las diferentes alternativas se encuentren en un rango muy cercano.

El modelo en sí tiene sus antecedentes en dos de las técnicas de toma de decisiones bajo incertidumbre más conocidas: Hurwicz y Laplace, y consiste en corregir el valor esperado, con la amplitud, a través de un parámetro beta $(\beta)$.

Para validar el modelo se probó con algunos problemas particulares en su mayoría, tomados de la literatura especializada, quedando sentada su aplicabilidad, pero a la vez se notó que el rango de variación del factor beta $(\beta)$, no pareciera tener sentido que fuese desde cero hasta uno, como se estableció en un principio, por lo cual como recomendación de dicha investigación se propuso, que se analizara con mayor profundidad el rango de variación de beta $(\beta)$.

Con este trabajo de investigación se presenta el resultado de este estudio, para el cual se siguió el método científico aplicado a la investigación de operaciones, donde primero se definió el problema, lo que se puede transcribir como los objetivos de la investigación: 
Analizar la variabilidad del factor beta $(\beta)$, en las aplicaciones de El Modelo de Amplitud (EMA), para que los resultados obtenidos con su aplicación no pierdan sentido físico.

En cuanto a las limitaciones y alcances, no se tiene otra que, analizar como las variaciones del factor $\beta$, pudiesen influir en la alternativa a tomar, cuidando a la vez que no se convierta éste en un parámetro a manipular, que permita obtener la decisión deseada y no la adecuada.

Como ya se ha indicado en otros trabajos, una premisa del grupo de investigación, es obtener productos que deben tener como característica su sencillez, de manera tal que no sea necesario un experto en modelos matemáticos, para su manejo.

\section{La toma de decisiones bajo incertidumbre y el modelo EMA}

En la toma de decisiones, en general, para cada estado de la naturaleza $(j)$ y para cada alternativa $(i)$ se produce un pago $\left(P_{i j}\right)$, los cuales son presentados a través de una matriz. En el caso particular de la toma de decisiones bajo incertidumbre, los estados de la naturaleza son conocidos pero no su probabilidad de ocurrencia $([1,3,5,6,7])$.

En la bibliografía especializada $([1,2,5,7,8,9,10,11,12])$ aparecen muchos modelos que permiten resolver los problemas bajo incertidumbre, pero los que se mencionan con mayor frecuencia son: Modelo Maximax, también conocido como el modelo del optimista, Modelo Maximin, conocido como modelo del pesimista o criterio de Wald, Modelo Minimax, o modelo del arrepentimiento, o de Savage, Modelo de realismo ([7]), mejor conocido como criterio de Hurwicz ([8]) y el Modelo del promedio ([7]), generalmente conocido como criterio de Laplace.

\subsection{El Modelo de Amplitud (EMA)}

El Modelo de Amplitud (EMA), tal como se describió en un trabajo anterior ([4]), es en si una combinación del modelo de Laplace, ya que lo primero que se hace es calcular los valores promedios, con el modelo de Hurwicz, dado que se corrige este valor promedio a través de un factor $\beta$, que actúa sobre la amplitud, funcionando ésta como un castigo para cada alternativa.

El sentido de El Modelo de Amplitud, y de allí la amplitud como castigo, es que entre alternativas con pagos similares, difiriendo en su medida de dispersión, debe ser seleccionada aquella que tenga menor dispersión, dado que implica un menor riesgo.

En resumen la expresión de El Modelo de Amplitud quedará, para el caso de maximización:

$$
\text { Alternativa }^{*}=\operatorname{Alternativa}(i) / \max _{i}\left\{V E_{i}+\left(\beta * V E_{i}-(1-\beta) * A_{i}\right)\right\}
$$

Con $A_{i}=$ mayor $P_{i j}$ - menor $P_{i j}$, como la amplitud de la alternativa $i$, y $V E_{i}$ el valor esperado de la alternativa i, calculado por el modelo de Laplace $V E_{i}=\sum_{j=1}^{n} P_{i j} / n$.

En el caso de minimización, manteniendo la misma nomenclatura se tiene:

$$
\text { Alternativa }^{*}=\operatorname{Alternativa}(i) / \min _{i}\left\{V E_{i}+\left(\beta * V E_{i}+(1-\beta) * A_{i}\right)\right\}
$$


En ambas ecuaciones (1) y (2), Alternativa* se refiere a la mejor alternativa.

Es importante destacar, que si bien, entre las medidas de dispersión disponibles se decidió usar la amplitud, El Modelo de Amplitud (EMA) no perdería validez si se usara la desviación estándar o la varianza.

\section{La sensibilidad del factor $\beta$}

Tal como se comentó anteriormente y se puede observar en las dos expresiones que se acaban de presentar, la medida de dispersión, la amplitud, se transforma en un elemento de castigo, restándose en el caso de maximización y sumándose en el caso de minimización.

También se puede notar que a medida que $\beta$ tiende a cero, se le da más peso a la amplitud, por lo cual se es más arriesgado, buscando mayores oportunidades de obtener valores extremos. Pero a medida que $\beta$ tiende a uno, se es más conservador, se le da menos peso a la amplitud y se tiende a tomar la decisión que genera el modelo de Laplace.

Como ya se dijo el valor de $\beta$, será el decisor quien lo fije y dependerá del problema en si y de su actitud ante el riesgo, pero para visualizar mejor esta situación y la aplicabilidad de EMA y en especial, los casos donde brinda un aporte relevante, se discutirán a continuación las posibles variaciones de $\beta$.

Para facilitar la ilustración, sin que esto signifique una perdida de generalidad, en todo este trabajo se usarán problemas de maximización, donde adicionalmente los valores esperados de las alternativas serán positivos.

\subsection{Sensibilidad de $\beta$ a través de métodos gráficos}

La primera aproximación al rango de variación del factor $\beta$, se hizo a través de métodos gráficos, para observar como podía variar la alternativa seleccionada de acuerdo al valor que se fijara de $\beta$, en problemas concretos. Para ello se usó el problema especial presentado en el trabajo anterior (Hernández y García 2001), que es un problema con tres alternativas (A1, A2 y A3) y tres estados de la naturaleza (E1, E2 y E3), cuyos pagos se transcriben en la tabla 1 , incluyendo en ella los valores esperados $\left(V E_{i}\right)$ y las amplitudes $\left(A_{i}\right)$ de cada una de las alternativas comparadas. Por razones de una mejor identificación se denominará a este problema HERGAR001.

Tabla 1: Tabla de pagos del problema HERGAR001.

\begin{tabular}{c|ccccc}
\hline \hline Alt. E.N. & E1 & E2 & E3 & $V E_{i}$ & $A_{i}$ \\
\hline A1 & 51 & 49 & 50 & 50 & 2 \\
A2 & 47 & 53 & 50 & 50 & 6 \\
A3 & 50 & 48 & 52 & 50 & 4 \\
\hline \hline
\end{tabular}

Partiendo de la ecuación para el caso de maximización (1), al tomar $\beta$ como factor común, se tiene:

$$
E M A i=\beta *\left(V E_{i}+A_{i}\right)+\left(V E_{i}-A_{i}\right)
$$


donde EMAi, representa el valor que tomaría la alternativa $i$, al aplicarle EMA. Por lo cual, para diferentes valores de $\beta$, la ecuación (3), representa una recta con corte en el origen igual a la diferencia entre el valor esperado y la amplitud, y pendiente la suma de estos dos valores. En la figura 1, se muestran las tres rectas que produce HERGAR001.

Figura 1: Sensibilidad de $\beta$ para el HERGAR001.

En la figura 1, se puede observar, que de acuerdo a los datos, para cualquier valor de $\beta$ comprendido entre cero y uno $(0<\beta<1)$, la alternativa 1 , con igual valor esperado que las restantes, pero con menor amplitud, se mantiene como la mejor, presentándose el empate cuando $\beta=1$, donde la amplitud no tiene ningún peso en la clasificación, y los resultados de EMA coinciden con Laplace.

Situación similar se puede observar en el HERGAR001A, que es una variación del problema anterior, en el cual los valores coincidentes son los de las amplitudes, mientras que los valores esperados difieren, tal como se representan en la tabla 2.

Tabla 2: Tabla de pagos del problema HERGAR001A.

\begin{tabular}{c|ccccc}
\hline \hline Alt. E.N. & E1 & E2 & E3 & $V E_{i}$ & $A_{i}$ \\
\hline A1 & 51 & 47 & 49 & 49 & 4 \\
A2 & 49 & 53 & 51 & 51 & 4 \\
A3 & 50 & 48 & 52 & 50 & 4 \\
\hline \hline
\end{tabular}

En la figura 2, similar a lo sucedido en la figura 1, hay una alternativa, desde el punto de vista de EMA, que es mejor a las demás, en este caso la alternativa dos (A2), que es la de mayor valor esperado, y como todas tienen igual amplitud, para cualquier valor de $\beta$ entre cero y uno $(0<\beta<1)$ se mantiene por encima.

Lo obtenido con el problema HERGAR001 y su variación HERGAR001A, lo que hace es comprobar la validez de EMA, pero no aporta mayor información acerca del rango de variación del parámetro $\beta$. Por ello se creó una segunda variación del HERGAR001, el HERGAR001B, en el cual una alternativa con mejor valor esperado, no es la de mejor amplitud, tal como se puede ver en los valores de la tabla 3. 
Figura 2: Sensibilidad de $\beta$ para el HERGAR001A.

Tabla 3: Tabla de pagos del problema HERGAR001B.

\begin{tabular}{c|ccccc}
\hline \hline Alt. E.N. & E1 & E2 & E3 & $V E_{i}$ & $A_{i}$ \\
\hline A1 & 50 & 49 & 48 & 49 & 2 \\
A2 & 49 & 55 & 49 & 51 & 6 \\
A3 & 48 & 50 & 52 & 50 & 4 \\
\hline \hline
\end{tabular}

Por su parte, la grafica que muestra los valores de la tabla 3, se representan en la figura 3 , donde se observa un punto de corte entre la alternativa uno y la alternativa dos, cuando $\beta$ toma un valor alrededor de 0,33 , es decir para $\beta$ menor a 0,3 se favorece la alternativa con menor valor esperado, pero con menor amplitud, mientras que para valores de $\beta$ mayores a 0,4 se favorece la alternativa con mayor amplitud, pero con mayor valor esperado, que sería la escogida si se hubiese usado directamente el método de Laplace.

Este último problema obliga a preguntarse, hasta donde se debería bajar el valor de $\beta$ sin que este favorezca demasiado a las alternativas de baja amplitud, aunque su valor esperado sea relativamente pobre, nótese que la alternativa tres, con un valor esperado y una amplitud intermedia, aunque se corta con cada una de las otras alternativas, siempre estuvo por debajo de alguna de ellas.

\subsection{Sensibilidad de $\beta$ a través de métodos analíticos}

Como a través de métodos gráficos sería muy tedioso ilustrar estas variaciones de amplitud, valor esperado y factor beta, a continuación se hará una revisión analítica de la situación. Para ello se partirá de nuevo de la ecuación anterior (3), y manteniendo las condiciones anteriores de valores esperados positivos y problemas de maximización, para dos alternativas diferentes se tendrá:

$$
\begin{aligned}
& \mathrm{EMA} 1=\beta *(V E 1+A 1)+(V E 1-A 1) \\
& \mathrm{EMA} 2=\beta *(V E 2+A 2)+(V E 2-A 2)
\end{aligned}
$$


Figura 3: Sensibilidad de $\beta$ para el HERGAR001B.

donde el 1 de EMA1, VE1 y A1 en la (4) y el 2 de EMA2, VE2 y A2 en la (5), representan a las alternativas uno y dos respectivamente, y para este caso se va a suponer que la alternativa uno es mejor, mayor, que la alternativa dos, por lo cual si se restan ambas ecuaciones (4) - (5) se tendrá:

$$
[(V E 1-V E 2)+\beta *(V E 1-V E 2)+(1-\beta) *(A 1-A 2)]>0
$$

Evidentemente la inecuación (6) será siempre positiva, y sólo será igual a cero en el caso que ambas alternativas sean iguales, es decir cuando el mismo $\beta$ produzca idénticos valores de El Modelo de Amplitud para ambas alternativas.

Si se despeja $\beta$ de esta última inecuación (6) se tendrá:

$$
\beta>-[(V E 1-V E 2)-(A 1-A 2)] /[(V E 1-V E 2)+(A 1-A 2)]
$$

Para la inecuación (7), que se convertirá en igualdad cuando las dos alternativas coincidan en EMA, se pueden analizar cuatro casos. Pero antes de analizarlos obsérvense las situaciones particulares, previamente comentadas, de valores coincidentes.

En primer lugar si $V E 1=V E 2$ y $A 1<A 2$, entonces $\beta>-1$, lo que se cumpliría siempre, en el rango de interés $0<\beta<1$, tal como se comprobó a través del HERGAR001 y la figura 1. En el caso de $A 1>A 2$, entonces cuando se hiciera el despeje desde la (6) se hubiese cambiado el signo de la desigualdad $\beta<-1$, lo que no tendría mucho sentido, pero nótese que se estaría contradiciendo la hipótesis que la alternativa uno es mejor que la dos.

En el caso de $A 1=A 2$ y $V E 1>V E 2$, entonces $\beta>-1$, que de nuevo se cumpliría siempre, en el rango de interés $0<\beta<1$, tal como se comprobó a través del HERGAR001A y la figura 2. Si por el contrario $V E 1<V E 2$, de nuevo cuando se hiciera el despeje en la (6) se hubiese cambiado el signo de la desigualdad $\beta<-1$, lo que tampoco tendría sentido, porque de nuevo se estaría contradiciendo la hipótesis que la alternativa uno es mejor que la dos.

Realizada las aclaratorias anteriores, se asumirá la siguiente nomenclatura para facilitar el análisis de los casos a estudiar $(V E 1-V E 2)$ de ahora en adelante se denominará $\Delta V E$ y $(A 1-A 2)$ se denominará $\Delta A$. 
Para el primer caso se tiene: $\Delta V E>0$ y $\Delta A>0$. En la primera variante de este caso (Caso 1a), $|\Delta V E|>|\Delta A|$ entonces quedaría el cociente de dos números mayores que cero, pero precedidos de un signo negativo, por lo cual $\beta<0$. Es decir la alternativa uno sería mejor que la alternativa dos incluso para valores de $\beta$ negativos, por lo cual lo será siempre en el rango de interés $0<\beta<1$.

Análisis similares se hicieron con los restantes casos, los cuales se resumen, junto a los que se acaban de revisar en la tabla 4, donde con un cambio* significa que en el rango de interés una alternativa es mejor hasta cierto valor de $\beta$ y luego la otra alternativa pasa a ser mejor; por otra parte si el resultado se cumple Siempre es que, en el rango, se respeta la hipótesis, para cualquier valor de $\beta$, e invertido, se debe interpretar que el resultado obtenido se cumple siempre, pero lo contrario a lo que se había establecido en la hipótesis, en el sentido que si se dijo que una alternativa era mejor que otra, lo que en realidad sucede es que esta segunda alternativa es mejor que la primera. Los casos de igualdad en los valores esperados se distinguieron como caso 0a y los de igualdad de amplitud como 0b, esto sólo para diferenciarlos de los casos donde hay diferencias entre los valores esperados y entre las amplitudes, los cuales, se debe esperar, representan mayor interés en el estudio de la variación de $\beta$.

Tabla 4: Resultados del análisis del $\beta$

\begin{tabular}{c|c|c|c}
\hline \hline Caso & Variante & $\beta$ & Resultado se cumple \\
\hline 0a.- $V E 1=V E 2$ & $0 a 1 .-A 1<A 2$ & $\beta>-1$ & Siempre \\
& $0 \mathrm{a} 2 .-A 1>A 2$ & $\beta<-1$ & Invertido \\
\hline 0b.- $A 1=A 2$ & 0b1.- $V E 1>V E 2$ & $\beta>-1$ & Siempre \\
& 0b2.- $V E 1<V E 2$ & $\beta<-1$ & Invertido \\
\hline 1.- $V E 1>V E 2$ y $A 1>A 2$ & 1a.- $|\Delta V E|>|\Delta A|$ & $-1<\beta<0$ & Siempre \\
& 1b.- $|\Delta V E|<|\Delta A|$ & $0<\beta<1$ & Con un cambio* \\
\hline 2.- $V E 1>V E 2$ y $A 1<A 2$ & 2a.- $|\Delta V E|>|\Delta A|$ & $\beta<-1$ & Siempre \\
& 2b.- $|\Delta V E|<|\Delta A|$ & $\beta>1$ & Siempre \\
\hline 3.- $V E 1<V E 2$ y $A 1>A 2$ & 3a.- $|\Delta V E|>|\Delta A|$ & $\beta<-1$ & Invertido \\
& 3b.- $|\Delta V E|<|\Delta A|$ & $\beta>1$ & Invertido \\
\hline 4.- $V E 1<V E 2$ y $A 1<A 2$ & 4a.- $|\Delta V E|>|\Delta A|$ & $-1<\beta<0$ & Invertido \\
& 4b.- $|\Delta V E|<|\Delta A|$ & $0<\beta<1$ & Con un cambio* \\
\hline \hline
\end{tabular}

Como se puede observar de la tabla 4, sólo los casos 1b, $V E 1>V E 2$ y $A 1>A 2$, con $|\Delta V E|<|\Delta A|$ y el 4 b, $V E 1<V E 2$ y $A 1<A 2$, con $|\Delta V E|<|\Delta A|$, producen un cambio de alternativa en el rango de interés, por lo cual serán estos dos casos los que se comentarán con mayor profundidad a continuación.

\subsection{Sensibilidad de $\beta$ a través de inspección numérica}

Aprovechando las ventajas que el ordenador brinda en estos tiempos, el último análisis de sensibilidad, se hará a través de una hoja Excel, donde se inspeccionarán algunos rangos 
de variación de los valores esperados y las amplitudes de dos alternativas, bajo los dos casos que resultaron de interés según el análisis anterior.

En la tabla 5, se refleja el caso $1 \mathrm{~b}$, donde como se comentó anteriormente y se puede observar en dicha tabla, el valor esperado y la amplitud de la primera alternativa son mayores que los de la segunda, pero la diferencia de amplitud supera la diferencia entre los valores esperados. En esta tabla se tienen los valores esperados ( $V E 1$ y $V E 2$ ) y las amplitudes ( $A 1$ y $A 2$ ), el valor de $\beta$ de intercepción tal como lo señala la igualdad correspondiente a la inecuación siete (7), seguidamente los valores de aplicar El Modelo de Amplitud a cada alternativa (EMA 1 y EMA 2), que como era de esperar, para cada caso resultan iguales. Para las tres últimas columnas se tiene Beta que es un valor de $\beta$ asignado y que se han tomado cero, cero punto cinco y uno, por ello cada caso se repite en tres filas, finalmente las dos columnas N EMA1 y N EMA2, que serían los resultados de aplicar, a la respectiva alternativa El Modelo de Amplitud, con el $\beta$ asignado.

Tabla 5: Valores para el caso $1 \mathrm{~b}$

\begin{tabular}{cc|cc|c|cc|c|cc}
\hline \hline VE1 & VE2 & A1 & A2 & $\beta$ & EMA 1 & EMA 2 & Beta & N EMA1 & N EMA2 \\
\hline 10 & 9 & 10 & 5 & 0,67 & 13,33 & 13,33 & 0,00 & 0,00 & 4,00 \\
10 & 9 & 10 & 5 & 0,67 & 13,33 & 13,33 & 0,50 & 10,00 & 11,00 \\
10 & 9 & 10 & 5 & 0,67 & 13,33 & 13,33 & 1,00 & 20,00 & 18,00 \\
100 & 95 & 100 & 50 & 0,82 & 163,64 & 163,64 & 0,00 & 0,00 & 45,00 \\
100 & 95 & 100 & 50 & 0,82 & 163,64 & 163,64 & 0,50 & 100,00 & 117,50 \\
100 & 95 & 100 & 50 & 0,82 & 163,64 & 163,64 & 1,00 & 200,00 & 190,00 \\
1000 & 999 & 10 & 5 & 0,67 & 1663,33 & 1663,33 & 0,00 & 990,00 & 994,00 \\
1000 & 999 & 10 & 5 & 0,67 & 1663,33 & 1663,33 & 0,50 & 1495,00 & 1496,00 \\
1000 & 999 & 10 & 5 & 0,67 & 1663,33 & 1663,33 & 1,00 & 2000,00 & 1998,00 \\
\hline \hline
\end{tabular}

A manera de ilustración se han presentado tres casos, donde los valores esperados están en el orden de las decenas, de las centenas y de las unidades de mil y las amplitudes en ordenes similares, excepto el tercer caso donde valores esperados del orden de las unidades de mil, tienen amplitudes del orden de las decenas.

Para todos ellos, tal como se analizó previamente $0<\beta<1$, pero nótese, viendo los valores de las columnas $\mathrm{N}$ EMA1 y N EMA2, que aunque la segunda alternativa empieza siendo mejor que la primera, cuando se supera el $\beta$ calculado, 0, 67 y 0,82 respectivamente, la primera alternativa pasa a ser mejor que la segunda.

Para el otro caso de interés (Caso 4b), en la tabla 6, siguiendo la misma identificación de las columnas que la tabla 5 , se muestran los resultados.

En esta tabla, como ya se había comentado, la primera alternativa tiene menor valor esperado y menor amplitud que la segunda, pero de nuevo la diferencia, en valor absoluto, entre las amplitudes es menor que la diferencia entre los valores esperados. De nuevo, observando las columnas correspondientes a los valores calculados (N EMA1 y N EMA2), hay un cambio de alternativa dominante, pero ahora es la alternativa uno la que es mejor 
para valores bajos de $\beta$ y luego superado el $\beta$ de intercepción, 0,67 ó 0,82, es la alternativa dos la superior.

Tabla 6: Valores para el caso 4b

\begin{tabular}{cc|cc|c|cc|c|cc}
\hline \hline VE1 & VE2 & A1 & A2 & $\beta$ & EMA 1 & EMA 2 & Beta & N EMA1 & N EMA2 \\
\hline 9 & 10 & 5 & 10 & 0,67 & 13,33 & 13,33 & 0,00 & 4,00 & 0,00 \\
9 & 10 & 5 & 10 & 0,67 & 13,33 & 13,33 & 0,50 & 11,00 & 10,00 \\
9 & 10 & 5 & 10 & 0,67 & 13,33 & 13,33 & 1,00 & 18,00 & 20,00 \\
99 & 100 & 90 & 100 & 0,82 & 163,64 & 163,64 & 0,00 & 9,00 & 0,00 \\
99 & 100 & 90 & 100 & 0,82 & 163,64 & 163,64 & 0,50 & 103,50 & 100,00 \\
99 & 100 & 90 & 100 & 0,82 & 163,64 & 163,64 & 1,00 & 198,00 & 200,00 \\
999 & 1000 & 5 & 10 & 0,67 & 1663,33 & 1663,33 & 0,00 & 994,00 & 990,00 \\
999 & 1000 & 5 & 10 & 0,67 & 1663,33 & 1663,33 & 0,50 & 1496,00 & 1495,00 \\
999 & 1000 & 5 & 10 & 0,67 & 1663,33 & 1663,33 & 1,00 & 1998,00 & 2000,00 \\
\hline \hline
\end{tabular}

Aunque no están reflejados en estas dos últimas tablas, es importante observar de ellas, como variaciones entre los valores esperados con una relación de uno a cinco, frente a las variaciones entre las alternativas, producen valores de $\beta(0,67)$, igual a lo que producen variaciones entre valores esperados con relación una a cincuenta con respecto a las variaciones de las alternativas.

Con estas últimas observaciones y dados todos los análisis anteriores, se pueden obtener algunas conclusiones.

\section{Conclusiones y recomendaciones}

La primera conclusión es reforzar lo ya dicho sobre El Modelo de Amplitud, y es su utilidad, ya que con los análisis aquí presentados queda mucho más clara su validez y campo de aplicación.

La segunda conclusión, que se puede obtener de los análisis anteriores, también es un refuerzo a lo dicho en el trabajo precedente, el rango de interés del factor $\beta$, debe reducirse a $0<\beta<1$, fuera de estos valores, se obtendrían resultados distorsionados, y en el caso particular de los extremos $\beta=0$, se le estaría dando igual peso al valor esperado que a la amplitud, lo que no tendría mucho sentido, y para $\beta=1$, se estaría aplicando el modelo de Laplace y no EMA.

En si el factor $\beta$, el cual debe depender del valor esperado de la alternativa y de su amplitud, queda demostrado que más que los valores absolutos de estos, lo importante es la relación que se guarde entre los primeros frente a las segundas:

1. Primeramente si una alternativa tiene mejor valor esperado y mejor amplitud, será seleccionada siempre, sin importar el valor de $\beta$ con el cual se trabaje (Casos $2 \mathrm{a}, 2 \mathrm{~b}$, 3a y $3 b)$. 
2. Por la misma razón frente alternativas de valores esperados iguales y diferentes amplitudes, siempre será seleccionada la de mejor amplitud, sin importar el $\beta$ escogido para el estudio (Problema HERGAR001).

3. De la misma manera, independiente del valor de $\beta$, entre alternativas de igual amplitud, pero diferentes valores esperados, será seleccionada la de mejor valor esperado (Problema HERGAR001A).

Analizando las afirmaciones del parágrafo anterior, sólo será interesante el valor de $\beta$, si la alternativa de mejor valor esperado, no es la de mejor amplitud (Problema HERGAR001B), para esta situación se tienen los siguientes casos:

1. Si la primera alternativa tiene mejor valor esperado, y peor amplitud, y la diferencia, en valores absolutos, entre los valores esperados es mayor que la diferencia entre las amplitudes, en el rango de interés, la alternativa uno será siempre mejor que la dos (Casos 1a y 4a).

2. Si la primera alternativa tiene mejor valor esperado, y peor amplitud, y la diferencia, en valor absoluto, entre los valores esperados es menor que la diferencia entre las amplitudes, en el rango de interés, la alternativa uno pasará de ser peor a ser mejor (Casos 1b y 4b). Estos últimos casos (1b y 4b), son los únicos, donde dependiendo del $\beta$, se seleccionará una u otra alternativa.

Otra conclusión interesante es el método de análisis utilizado, que es una mezcla de métodos analíticos, con gráficos e inspección numérica, los cuales se refuerzan unos a otros, para facilitar llegar a conclusiones adecuadas.

También es importante concluir acerca del valor de cambio del $\beta$, el cual no parece depender del porcentaje de variación entre los valores esperados, ni entre las alternativas, ni siquiera entre las relaciones entre estas variaciones.

Estas dos últimas conclusiones permiten sugerir algunas recomendaciones, siendo la primera de ellas que se analice con mayor profundidad el uso conjunto de los métodos utilizados aquí para aplicarlos a investigaciones donde se deban comparar comportamientos de modelos matemáticos.

La siguiente recomendación es seguir profundizando el estudio de sensibilidad del factor $\beta$, para ver si existe alguna relación que permita saber a priori que valor de $\beta$ es recomendable utilizar en un determinado análisis de alternativas.

Finalmente se recomienda seguir profundizando el estudio de El Modelo de Amplitud (EMA), recomendándose la creación de problemas con situaciones particulares, que permitan visualizar una mejor aplicabilidad del mismo.

\section{Agradecimientos}

Este trabajo no hubiese sido posible sin el apoyo brindado por la Universidad Metropolitana, en especial el decanato de Investigación y Desarrollo, y el Decanato de Ingeniería, a través de la Escuela de Ingeniería de Sistemas, y el de Minimax Consultores, C.A., en especial su Gerencia de Investigación. 


\section{Referencias}

[1] Baumol, W.J. (1980) Teoría Económica y Análisis de Operaciones. Prentice Hall, Bogotá.

[2] Del Valle, J.A. (2001) "Introducción a la teoría de decisiones", en: http://members.es.tripo.de/JAVICA/Decisiones/Intro.Decisiones.html. Consultada (10/04/2001).

[3] El proceso (2001) "El proceso de toma de decisiones", en: http://www .monografias.com/trabajos4/orgad/orga2. shtml. Consultada (11/04/2001).

[4] Hernández R., J.G.; García G., M.J. (2001) "Toma de decisiones bajo incertidumbre considerando la dispersión", en: XXVII Conferencia Latinoamericana de Informática, Mérida, Venezuela.

[5] Hillier, F.S.; Lieberman, G. (1974) Operations Research (2a. ed). Holden-Day, Inc., San Francisco.

[6] León, O.G. (2001) Tomar Decisiones Difíciles (2a ed). McGraw-Hill, Madrid.

[7] Levin, R.I.; Kirkpatrick, C.A. (1985) Enfoques Cuantitativos a la Administración. Compañía Editorial Continental, México.

[8] Mathur, K.; Solow, D. (1996) Investigación de Operaciones, el Arte de la Toma de Decisiones. Prentice Hall, México.

[9] Rheault, J.P. (1982) Introducción a la Teoría de Decisiones con Aplicaciones a la Administración. Limusa, México.

[10] Taha, H. A. (1998) Investigación de Operaciones una Introducción (6a ed). Prentice Hall, México.

[11] Toma (2001) "Toma de decisiones", en: http://yupana. autonoma.edu.co/di/administracion/tema6.html. Consultada: (18/03/2001).

[12] Winston, W. (1994) Investigación de Operaciones: Aplicaciones y Algoritmos. México: Grupo Editorial Iberoamérica. 